\title{
Análisis geográfico y conservación del zorro andino Lycalopex culpaeus (Mammalia, Canidae) en Colombia
}

\author{
Elkin A. Noguera-Urbano ${ }^{1,2}$, Héctor E. Ramírez-Chaves ${ }^{3}$ \& María M. Torres-Martínez ${ }^{4}$
}

\begin{abstract}
1. Grupo de Investigación de Biogeografía de la Conservación, Posgrado en Ciencias Biológicas, Departamento de Biología Evolutiva, Facultad de Ciencias, Universidad Nacional Autónoma de México, Ciudad de México, México. (elkalexno@gmail.com)

2. Grupo de Investigación de Ecología Evolutiva, Programa de Biología, Universidad de Nariño, Ciudadela Universitaria Torobajo, Pasto, Colombia.

3. School of Biological Sciences, University of Queensland, Goddard Building 8, St. Lucia 4072, Brisbane, Australia. (hera.chaves@gmail.com)

4. Laboratório de Biodiversidade, Conservação e Ecologia de Animais Silvestres, Programa de Pós-graduação em Ecologia e Conservação, Universidade Federal

do Paraná, Curitiba, Paraná, Brasil. (canasmarianita@gmail.com)
\end{abstract}

Recibido 6 Septiembre 2015.

Aceptado 6 Junio 2016

DOI: $10.1590 / 1678-4766 e 2016014$

\begin{abstract}
Geographical analysis and conservation of the Andean fox Lycalopex culpaeus (Mammalia, Canidae) in Colombia. The Andean fox Lycalopex culpaeus (Molina, 1782) has been included in the category of threat Vulnerable in Colombia, however, there are not available studies that support at the national level the inclusion of this species in that category. In this work, we delimited the distribution area and we discuss the category of threat of the species in Colombia. For this, we used a niche modelling approach using the Maximun Entropy (Maxent) algorithm. Using Geographical Information Systems, we highlighted areas in which the species is protected. Our results show that the Andean fox can be found potentially from Nariño until Huila departments $\left(8,877 \mathrm{~km}^{2}\right)$, and the National System of Protected Areas in Colombia protected $1,742 \mathrm{~km}^{2}(19.6 \%)$ of the potential distribution of the species. Despite the species is considered Vulnerable in Colombia, we found that it does not meet the criteria for allocation in that category, due to the absence of tangible evidence that supported a population decline, decreased in area of occupancy, or habitat quality in the decade prior to the risk assessment. Considering the validated records and information on the distribution area of the species in Colombia, we suggest a re-assignation of the category to the category Near Threatened (NT).
\end{abstract}

KEYWORDS. Andes, distribution, vulnerability, niche modelling.

RESUMEN. El zorro andino Lycalopex culpaeus (Molina, 1782) ha sido incluido en la categoría de amenaza Vulnerable en Colombia, sin embargo, no se han realizado estudios que validen la inclusión de la especie en dicha categoría a nivel nacional. En el presente trabajo se delimitó el área de distribución potencial (DP) de la especie en Colombia y se discute su categoría de amenaza. Para ello se usó modelamiento de nicho ecológico en el algoritmo de Máxima Entropía (Maxent). Con procesamiento en Sistemas de Información Geográfico resaltamos las áreas donde la especie se encuentra protegida. Los resultados muestran que el zorro andino se podría encontrar desde los departamentos de Nariño hasta el Huila $\left(8,877 \mathrm{~km}^{2}\right)$, y el $19.6 \%(1,742 \mathrm{~km})^{2}$ del área de distribución potencial de la especie se encuentra dentro del Sistema Nacional de Áreas Protegidas de Colombia. A pesar de que la especie es considerada Vulnerable en Colombia, se encontró que no cumple con los criterios para su asignación en dicha categoría debido a la ausencia de evidencias tangibles que sustenten una reducción poblacional, disminución del área de ocupación o la calidad del hábitat en la década previa a la asignación de la categoría de amenaza. Teniendo en cuenta los registros verificables e información sobre el área de distribución de la especie en Colombia, se propone una reasignación de la categoría de amenaza nacional a Casi Amenazada (NT).

PALABRAS-CLAVE. Andes, distribución, vulnerabilidad, modelamiento de nicho.

El zorro andino o lobo de páramo (Lycalopex culpaeus) es una de las cinco especies de Canidae presentes en Colombia (SolARI et al., 2013), habita en zonas de páramos y bosque andino entre los 2,000 y 3,700 msnm (JORGENSON et al., 2006). Históricamente, la especie ha sido incluida dentro del género Pseudalopex Burmeister, 1856, aunque ambos nombres son sinónimos, y Lycalopex Burmeister, 1854 tiene prioridad ya que fue erigido dos años antes (ZunINo et al., 1995).

Inicialmente se consideró que en Colombia la especie se distribuía desde los departamentos de Risaralda y Tolima hasta Nariño en el suroccidente del país (JORGENSON et al., 2006). Sin embargo, desde el primer registro de la especie para Colombia con base en un ejemplar recolectado en Nariño
(HERShKovitz, 1957), son pocos los reportes adicionales conocidos. En colecciones biológicas se han localizado sólo dos ejemplares con localidad precisa (RAMÍREZ-CHAVES et al., 2013), y otro ejemplar sin lugar de captura definido (SuÁreZ-Castro \& Ramírez-ChaVes, 2015). Otros registros recientes provienen de fotografías y entrevistas (RAMÍREZChaves \& Noguera-Urbano, 2010; Ramírez-Chaves et al., 2013) obtenidas en diferentes localidades en el Nudo de Los Pastos, en el departamento de Nariño. La subespecie que se encuentra en Colombia es L. culpaeus reissii Hilzheimer, 1906, la cual fue descrita a partir de ejemplares procedentes de Quito, Ecuador (HershKovitz, 1957), y cuya distribución comprende los Andes de Ecuador y suroccidente de Colombia (Sillero-Zubiri, 2009). 
Debido a los pocos registros de presencia, hasta el momento hay incertidumbre sobre la distribución del zorro andino en Colombia. Muchos de los registros en literatura fuera de Nariño (e. g. JoRGENSON et al., 2006) han sido definidos como dudosos o erróneos por la ausencia de ejemplares (RAmírez-Chaves et al., 2013). Por esto, la especie ha sido restringida al suroccidente de Colombia en el Nudo de los Pastos, departamento de Nariño (JIMÉNEZ et al., 2008; Ramírez-Chaves \& Noguera-Urbano, 2010; RAMÍREZ-CHAVES et al., 2013).

Por otra parte, las poblaciones de zorro andino en Colombia han sido incluidas en la categoría de amenaza Vulnerable - VU, de acuerdo a la Resolución 0192 de 2014 del Ministerio de Ambiente y Desarrollo Territorial (MADT, 2014). Sin embargo, en países limítrofes como Ecuador y Perú, así como a nivel global no se encuentra amenazada, por lo que ha sido categorizada como Preocupación Menor - LC (JimÉnez et al., 2008; Tirira, 2011; Cossíos et al., 2012). El criterio para la inclusión de la especie en la categoría VU a nivel nacional, con base en información disponible hasta el año 2004, fue la rápida disminución poblacional debido a la transformación de hábitat y la cacería de retaliación por depredación de ganado (JorGENSON et al., 2006). Sin embargo, las fuentes de dicha información no son claras ya que no existen estudios relacionados con estos temas en Colombia (ver SuÁREZ-CASTRo et al., 2015), y en general es poco lo que se conoce sobre la especie en el país. A partir de registros recientes se ha sugerido que la categoría de amenaza nacional debería reconsiderarse (RAMíREZ-CHAVEs et al., 2013). También, la ausencia de información complementaria o estudios que soporten la reducción poblacional de la especie generan dudas sobre su correcta inclusión en la categoría VU.

Las circunstancias antes descritas hacen necesaria una reevaluación del área de distribución y del grado de amenaza de la especie a nivel nacional. Una de las alternativas que permite proponer y hacer inferencias sobre la distribución de especies y otros temas asociados, es el modelamiento de nicho ecológico (Peterson et al., 2011; AraúJo \& Peterson, 2012). Con las técnicas de modelamiento es posible determinar mapas de distribución potencial (DP) y luego emplearlos en propuestas de estrategias de conservación y la verificación del estado ecológico de especies carismáticas (Rodríguez-Soto et al., 2011; De CAStro Pena et al., 2014; Morato et al., 2014; ORTEGA-ANDRADE et al., 2015). Los objetivos de este estudio son actualizar la distribución geográfica y clarificar aspectos relacionados al estatus de amenaza del zorro andino en Colombia. Además, se presenta un nuevo registro marginal para la especie, que en conjunto con la información obtenida sobre la distribución permitió sugerir una recategorización del zorro andino en Colombia, y se discute la ausencia de evidencias asociadas a la información empleada para establecer la categoría de amenaza en trabajos previos.

\section{MATERIALES Y MÉTODOS}

Localidades de presencia. La obtención de localidades para Colombia y Ecuador se realizó a partir de la consulta de la red de Mammal Networked Information System (MANIS; http://manisnet.org/), de registros obtenidos en campo por los autores, fuentes bibliográficas en las que se reportan avistamientos de la especie (RAMÍREZ-CHAVES \& Noguera-Urbano, 2010; Ramírez-Chaves et al., 2013; SuÁREZ-CASTRo \& RAmíreZ-ChaVES, 2015), y un nuevo registro fotográfico procedente de la Reserva Natural del Azufral, municipio de Túquerres, Nariño (Febrero 19 de 2013, 1N 9'40"- 77W41'8"). Algunas localidades sin georreferencia fueron asignadas consultando Google Earth (https://www. google.es/intl/es/earth/index.html). Todas las localidades y datos geográficos fueron verificadas en Google Earth para detectar y eliminar errores (CUYCKENS et al., 2015; ORTEGAANDRADE et al., 2015). Las localidades fueron almacenadas como grados decimales en el sistema WGS84. El área de vida del zorro andino se encuentra entre $0,72 \mathrm{ind} / \mathrm{km}^{2}$ y $1,3 \mathrm{ind} /$ $\mathrm{km}^{2}$ (Novaro, 1997), así que en un programa de Sistemas de Información Geográfica (SIG) se aplicó un filtro con distancia de $2 \mathrm{~km}^{2}$ para disminuir la densidad de localidades.

Modelo de nicho ecológico y distribución de la especie. Se generó un modelo de la distribución para el zorro andino en Colombia y Ecuador usando el Algoritmo de Máxima Entropía (PHILLIPS et al., 2006, 2008) implementado en el programa Maxent 3.3.3k (https://www.cs.princeton. edu/ schapire/maxent/). El programa calcula la probabilidad de distribución o idoneidad ambiental de la especie con base en los datos de presencia y variables ambientales sobre los pixeles de un área modelada, considerando que la mejor solución se obtiene al maximizar la entropía de dicha distribución bajo ciertas condiciones ambientales (PHILLIPS et al., 2006). En Maxent se obtuvo un modelo con el 60\% de las localidades (entrenamiento), mientras que el $40 \%$ se usó para evaluación externa. Se activó la opción de semilla aleatoria "Random seed", sin extrapolar "No extrapolate" y con salida logística "Output format: Logistic", las otras opciones se mantuvieron por defecto "default". El área de movilidad (área potencialmente visitada por la especie) debe ser tenida en cuenta para la construcción del modelo (BARVE et al., 2011), esta fue definida extrayendo de la propuesta de ecorregiones de OLson et al. (2001). Se incluyeron las categorías de Bosques Montanos Andinos Noroccidentales, Bosques Montanos de la Cordillera Oriental y los Páramos de los Andes del Norte ubicados sobre ambas ecorregiones, desde los $3^{\circ}$ Norte hasta los $6^{\circ}$ Sur. El área de movilidad seleccionada representa los ambientes de tolerancia, zonas de interacción biótica y barreras de dispersión descritos para la especie (Novaro, 1997; JorGENSON et al., 2006). En Maxent se incorporaron las variables bioclimáticas a resolución aprox. de $1 \mathrm{~km}^{2}$ (Humans et al., 2005), se seleccionaron las que tienen importancia biológica para la especie, por ejemplo la distribución del zorro andino se ha relacionado con la temperatura media de las mínimas del mes más frío e indirectamente con la precipitación media anual (disponibilidad y cantidad de agua en humedales), debido a que usa a los humedales como sitios de caza (CUYCKENs et al., 2015). Además se incluyeron las variables con los mayores porcentajes de contribución obtenidos de una prueba de 
"Jackknife" de un ensayo inicial en Maxent y aquellas con la menor multicolinealidad. La multicolinealidad se disminuyó a partir de un análisis de agrupamiento de variables basado en una matriz de correlación, en cada grupo se descartaron variables a partir de los factores de inflación de varianza (VIF>3: r=0.8; ZuUR et al., 2010). Los procesamientos estadísticos fueron realizados con herramientas de los paquetes Raster (HiJMans et al., 2005) y HH (HeIBERGER, 2015) en el programa $R$ ( $R$ DeVELOPMENT CORE TEAm, 2014). Las variables bioclimáticas seleccionadas fueron recortadas con un mapa del área de movilidad para ser ingresadas en Maxent. En la evaluación del modelo se consideraron los valores del Área Bajo la Curva (AUC) y la Característica Operativa del Receptor (ROC), respectivamente miden el ajuste del modelo y la capacidad de predicción del modelo versus el azar (HANLeY \& MCNeIL, 1982). El AUC ha sido criticado por dar peso similar a la omisión y comisión, y ocasionar errores en la selección de los modelos (PETERSON et al., 2008). Por lo tanto se calcularon la ROC parcial y AUCratio (PETERSON et al., 2008) en el programa Tool for Partial-ROC 1.0 (BARVE, 2008) con el 40\% restante de localidades. Se aplicaron 1000 iteraciones y se consideró el $5 \%$ de error (E). La significancia del AUCratio fue estimada con Bootstrapping (1000 réplicas) de 13 localidades (50\% de las localidades de entrenamiento) y cálculo de sus AUCratios (Peterson et al., 2008). Cuando el 95\% de las réplicas de AUCratios obtenidas con Bootstrapping fueron mayores que uno (AUCratios>1) se rechazó la hipótesis nula de que el rendimiento no es mejor que lo esperado por el azar (Peterson, 2012).

Análisis geográfico del zorro andino en Colombia. El nicho ecológico fue explorado en dos gráficos de tres dimensiones: (a) Probabilidad versus altitud y heterogeneidad ambiental (HA). El mapa de HA fue obtenido a partir de un análisis de componentes principales de las variables ambientales empleadas para el modelo, valores cercanos a 1 indican sitios de mayor HA. (b) Probabilidad versus precipitación anual y temperatura anual media. A partir del modelo se estimó un mapa de costo de dispersión, con el fin de refinarlo quitando las áreas que posiblemente son inaccesibles para la especie. Los valores invertidos del modelo pueden ser considerados como mapas de "costo de dispersión", las zonas con altas probabilidades tendrán bajo costo a la dispersión mientras que zonas con bajas probabilidades tienen alto costo a la dispersión (CHAN et al., 2011). El modelo refinado con valores de formato logístico fue transformado a modelo de distribución potencial (DP; presencia potencial $=1$ y ausencia $=0$ ) tomando como umbral de corte el valor mínimo de probabilidad de las localidades de entrenamiento ("Minimum training presence"; ANDERSON \& GoNZÁLEZ, 2011). Este umbral incluye todas las localidades de entrenamiento usadas para la obtención del modelo, considerando que cada una de ellas representa el hábitat de la especie. Se determinó el área habitable (AH) para el zorro andino, para ello se obtuvo un "raster" de la diferencia de la DP menos el área disponible (AD). El área disponible fue extraída de una imagen reclasificada de uso de suelo (Época 2008-2012; Defourny \& VAN Bogaert, 2014). La reclasificación se realizó teniendo en cuenta descripciones de hábitat para la especie (NOVARO, 1997): se consideraron seis categorías como área disponible (árboles, árboles y arbustos siempre verdes $>15 \%$, árboles y arbustos deciduos $<15 \%$, herbáceas, mosaico árboles-arbustos-hierbas, matorrales) y nueve categorías como área no disponible (agua, cuerpos de agua, cultivos, mosaico cultivos-vegetación natural $<50 \%$, mosaico vegetación natural-cultivos $<50 \%$, pantanos, pastos, suelos desnudos, zonas urbanas). Se cuantificó la extensión de la DP y el AH del zorro andino en Colombia contabilizando el número de pixeles dentro de un polígono del país. Las dos áreas se estimaron a escala de departamento, considerando que las medidas de conservación se toman por unidades políticas. Para ello se intersectaron los mapas de la DP y AH con un mapa político administrativo de Colombia y se contabilizaron el número de pixeles incluidos en cada departamento. Además, se evaluó la inclusión del área de distribución del zorro andino dentro del Sistema de Nacional de Áreas Protegidas (SINAP; incluye Parques Naturales, Reservas Forestales, Reservas de la Sociedad Civil, Santuarios de Flora y Fauna, entre otras), para ello se intersectaron los mapas de la DP y AH con un mapa del SiNAP (Parques Nacionales Naturales de Colombia, 2014), se obtuvo el número de pixeles en el total de las áreas. El área de distribución del zorro andino contenida dentro de cada unidad de comparación (País, departamento, Área protegida según SINAP) fue estimada a partir del producto del número de pixeles por $1 \mathrm{~km}^{2}$ (resolución de cada pixel).

Reevaluación de la categoría de amenaza en

Colombia. La reevaluación de la categoría se realizó con base en los criterios y directrices expuestos por la Unión Internacional para la Conservación de la Naturaleza - UICN (UICN, 2012a, b). Entre los criterios empleados para incluir a una especie en la categoría de amenaza VU, en la cual ha sido asignada $L$. culpaeus en Colombia, se encuentran: (A) reducción del tamaño de la población; (B) distribución del tamaño de la población; (C) tamaño de la población estimada en menos de 10.000 individuos maduros; (D) población muy pequeña o restringida y (E) probabilidad de extinción en estado de vida silvestre de al menos un $10 \%$ dentro de 100 años a partir de datos cualitativos. Teniendo en cuenta dichos criterios y a partir de información disponible en literatura (JORGENSON et al., 2006; RAMírez-CHAVES et al., 2013), observaciones recientes de la especie en Colombia por parte de los autores y los datos obtenidos del modelo de distribución generado en este trabajo, se revisó si la especie debe ser categorizada como VU a nivel nacional.

\section{RESULTADOS}

Modelo de distribución de la especie. Se emplearon 44 localidades (26 localidades para el entrenamiento del modelo, Apéndice 1) y nueve variables bioclimáticas que aportaron con porcentajes diferentes (Tab. I) en la construcción del MNE con Maxent. El modelo presentó un AUC de entrenamiento (0.96) y AUCratio (1.4 \pm 0.3$)$ 
Tab. I. Variables bioclimáticas y sus porcentajes de contribución en la construcción del modelo de distribución del zorro andino. La contribución fue estimada con la prueba de "Jackknife" realizada por Maxent. La isotermalidad (Bio3) y la temperatura estacional (Bio4) son usadas para calcular la variable Bio5.

\begin{tabular}{lcc}
\hline Variable & Nombre de la variable & Porcentaje de contribución \\
\hline Bio2 & Media del intervalo de temperatura diario & 6.5 \\
Bio4 & Temperatura media de las mínimas del mes más frío & 6.2 \\
Bio5 & Intervalo anual calculado como Bio3-Bio4 & 58.7 \\
Bio12 & Precipitación (P) media anual & 15.6 \\
Bio14 & Precipitación media del mes más seco & 5 \\
Bio15 & Precipitación estacional (coeficiente de variación) & 1.3 \\
Bio17 & Precipitación total media del cuatrimestre más seco & 1.2 \\
Bio18 & Precipitación total media del cuatrimestre más cálido & 1.1 \\
Bio19 & Precipitación total media del cuatrimestre más frío & 4.4 \\
\hline
\end{tabular}

significativo $(\mathrm{p}<0.05$; AUCratio $=1.8 \pm 0.1)$; el umbral logístico seleccionado "Minimum training presence" fue 0.16 .

Análisis geográfico del zorro andino en Colombia. Los sitios con mayor probabilidad de ocurrencia del zorro andino se encuentran en las montañas andinas sobre los $2.400 \mathrm{~m}$ de altitud y son ambientalmente estables (HA cercana a cero; Fig. 1), con precipitaciones cercanas a los $1.000 \mathrm{~mm} / \mathrm{año} \mathrm{y}$ temperaturas promedio anuales sobre $\operatorname{los} 6^{\circ} \mathrm{C}$ (Fig. 2). Los mapas de probabilidades y costo de dispersión mostraron dos fragmentos de la distribución, que se mantuvieron aislados por áreas de baja probabilidad (Fig. 3) y alto costo de dispersión (cercano a 1; Fig. 4): el fragmento "a" se ubicó al norte del cañón del río Patía (Nariño: Policarpa, Colombia) y el fragmento "b" se encontró en los páramos Los Picachos (cordillera Oriental: entre los departamentos de Huila y Caquetá, Colombia). Considerando el mapa de costo de dispersión y la ausencia de registros verificables del zorro andino en los dos fragmentos (RAMíREZ-CHAVES et al., 2013), ambos fueron removidos durante el refinamiento del modelo para la obtención del mapa de DP. El zorro andino (Fig. 5) se distribuye potencialmente en área de $41,810 \mathrm{~km}^{2}$, desde el sur del Ecuador hasta el centro de la cordillera Occidental colombiana. En Colombia el zorro andino (Fig. 6) se distribuye potencialmente desde Nariño hasta el páramo de las Papas en el Huila $\left(14,600 \mathrm{~km}^{2}\right.$; Fig. 7 ), incluyendo territorios en Putumayo y Cauca. En total la DP cubre el $0.69 \%$ de la extensión de Colombia (Tab. II). El AH para el zorro andino en Colombia es de $8,877 \mathrm{~km}^{2}$ (Fig. 8), la pérdida con respecto al DP fue del 39.2\% (Fig.

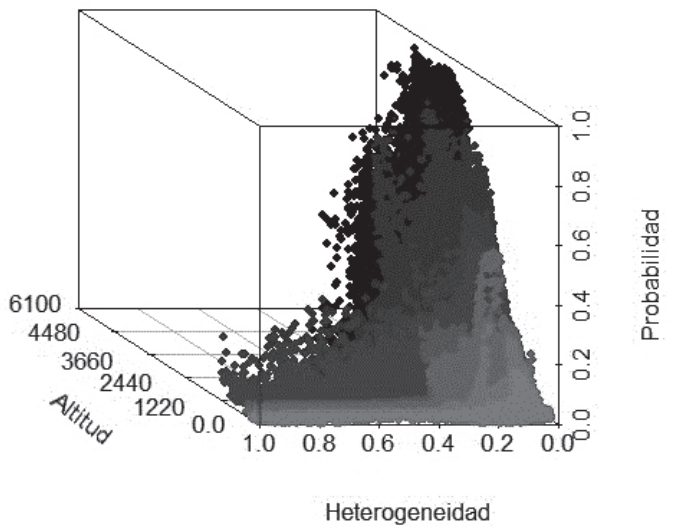

Fig. 1. Distribución de la probabilidad del modelo de Maxent con respecto a la altitud/heterogeneidad ambiental.
9). A escala nacional se encuentran $1.742 \mathrm{~km}^{2}$ (19.6\%) del AH dentro del SINAP (Fig. 8; Tab. II). La AH de la especie se concentra en Nariño (65.4\%; Tab. II), así como también la mayor pérdida de área (47\%), mientras que tan solo se conserva el $8.7 \%$ en el SINAP (Tab. II). La menor AH para el zorro andino se encuentra en el Putumayo $\left(321 \mathrm{~km}^{2}\right)$, por otra parte la mayor inclusión dentro del SINAP (67.5\%) y la menor pérdida de área (15.9\%) ocurren en el Huila (Fig. 9).

Reevaluación de la categoría de amenaza en Colombia. En la evaluación anterior (JORGENSON et al., 2006) L. culpaeus fue catalogada como VU A2cd+3cd para Colombia. Para que la especie se pueda incluir dentro de dicha categoría se requiere de una reducción de tamaño de la población (estimada, inferida, observada, proyectada o sospechada) en los últimos 10 años, donde las causas de la reducción no hayan cesado. Esta reducción debe estar apoyada en la disminución del área de ocupación o de la calidad de hábitat para la especie, y de explotación real o potencial de la misma (UICN, 2012a). A nivel nacional, no existen estudios poblacionales que soporten la inclusión de la especie en la categoría VU, aunque los subcriterios para sustentar la categoría pueden basarse en una inferencia o sospecha de reducción de un 30\% del tamaño de la población. Sin embargo, el nuevo registro fotográfico (Fig. 5), así como las entrevistas realizadas en el departamento de Nariño (RAMíREZ-CHAVEs et al., 2013), sugieren que la especie es común en zonas montañosas conservadas, zonas intervenidas y algunas áreas protegidas del departamento, y que la única presión que sufre es por cacería de retaliación.

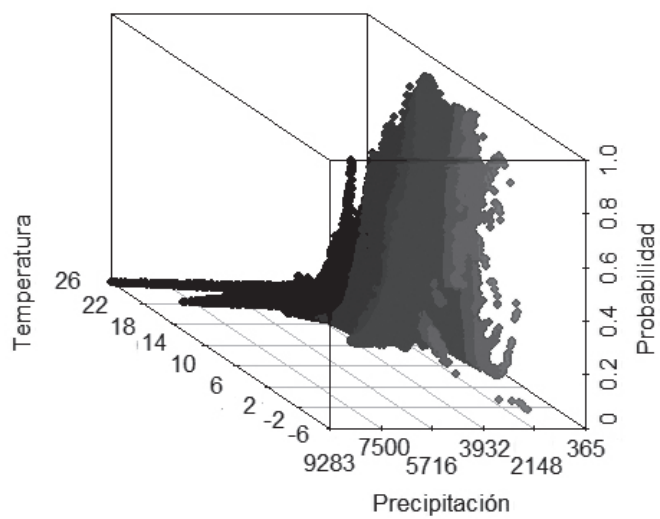

Fig. 2. Probabilidad con respecto a la precipitación anual/temperatura anual media. 

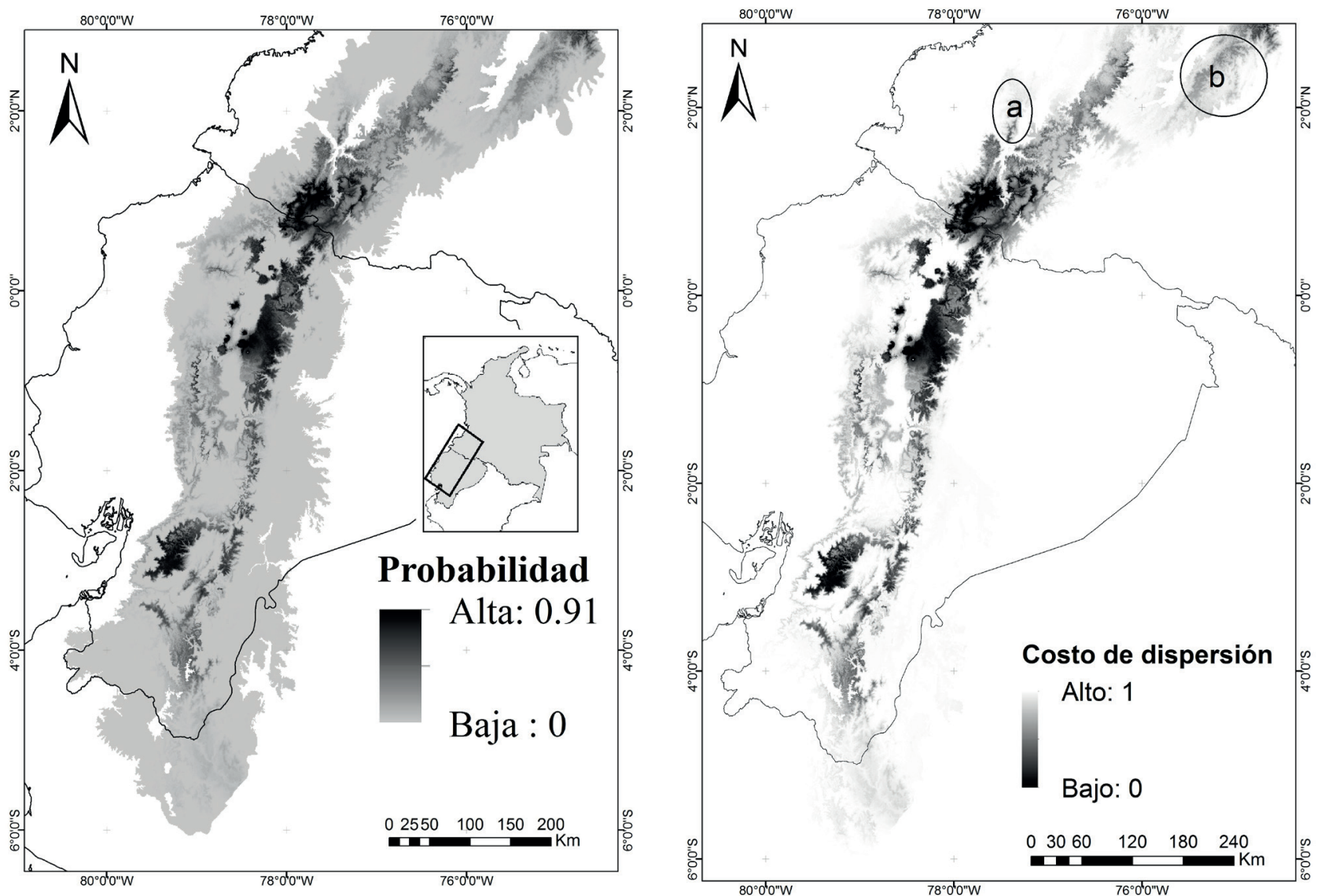

Figs 3-4. Modelo de distribución potencial del zorro andino: Fig. 3, mapa con probabilidades logísticas y mapa de costo de dispersión; Fig. 4, fragmentos (a y b) removidos para la obtención del mapa binario de distribución potencial.

Considerando el modelo de distribución potencial se observa que hay continuidad entre zonas con ambientes idóneos para el zorro andino, así que la especie probablemente podría contar con áreas de tamaño adecuadas para su mantenimiento a futuro, y con pocas (o ninguna) barreras geográficas que bloqueen el flujo de individuos entre Ecuador (donde la especie se encuentra categorizada como Preocupación Menor) y Colombia. Esto último es un criterio que se debe tener en cuenta cuando se pretende realizar una categorización regional diferente a la global para una especie (UICN, 2012b). Todas las situaciones antes mencionadas sugieren que el zorro andino no cumple los requerimientos para ser tratada dentro de las categorías de amenaza $\mathrm{VU}$ en el país, o en otras categorías mayores (En Peligro Crítico o En Peligro), por tanto, la transferencia de una categoría de amenaza mayor a una categoría de amenaza menor puede efectuarse (UICN, 2012b). En este caso se considera que la especie debe incluirse en la categoría Casi Amenazada (NT) a nivel nacional.

\section{DISCUSIÓN}

Nuestros resultados confirma que el zorro andino en Colombia y Ecuador se encuentra restringido a ecosistemas de altamontaña (Bosque Andino, Bosque Altoandino, Subpáramo y Páramo), como había sido sugerido por otros autores (Jorgenson et al., 2006; Trujillo \& Trujillo, 2007). Además, la especie presenta un nicho ecológico relacionado con bajas temperaturas, con precipitaciones desde bajas hasta medias y ambiente estables, lo cual corresponde con las descripciones de hábitat realizadas para la especie en el Ecuador (Trujillo \& Trujillo, 2007). En contraste las poblaciones septentrionales del zorro andino (Perú hasta Argentina) habitan en zonas con ámbitos ambientales amplios, ya que en países como Perú y Chile se los encuentra en zonas boscosas, desérticas y hasta semiáridas (JIMÉNEZ et al., 2008). En cuanto a la distribución conocida de la especie en Colombia, recientemente se ha propuesto que sus poblaciones habitan el suroccidente del país, principalmente en Nariño y Putumayo (Ramírez-Chaves et al., 2013; SuÁRez-CaStro et al., 2015). De acuerdo con la UICN la distribución de la especie en Colombia cubre una extensión aproximada de 6,161 $\mathrm{km}^{2}$ (JIMÉNEZ et al., 2008), áreas que sería aproximadamente la mitad de la sugerida en este estudio $\left(14,600 \mathrm{~km}^{2}\right)$. Eso se debe a que la UICN restringió la distribución de la especie a la porción suroccidental del departamento de Nariño, ya que, cuando se formuló dicho mapa de distribución no se contaba con otros registros verificados en el noroccidente del departamento (RAMÍrez-CHAVEs et al., 2013), o las menciones realizadas en el Putumayo (Jorgenson et al., 2006). L o s resultados soportan la sugerencia que la distribución de la especie en Colombia se extiende desde Nariño hasta el 

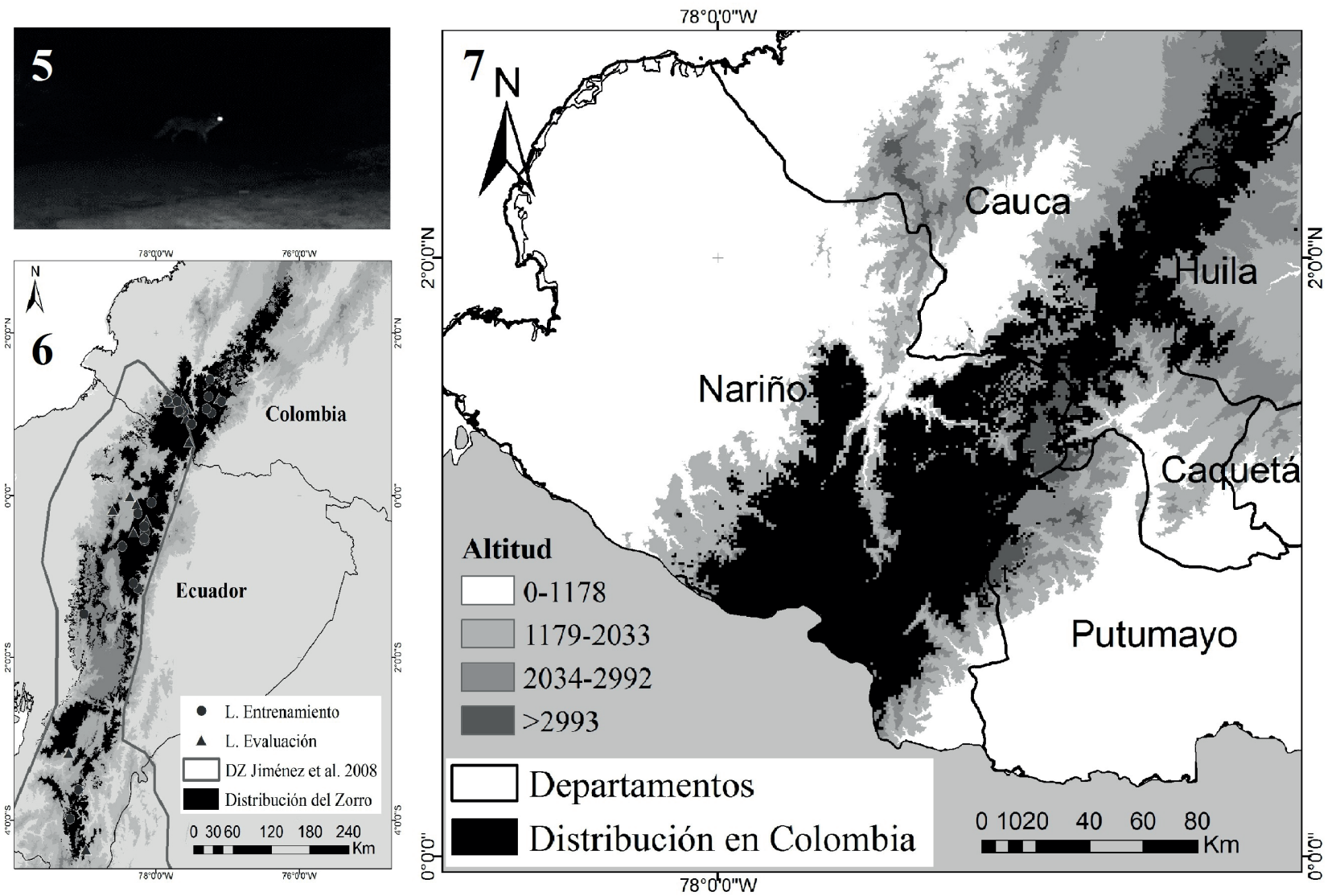

Figs 5-7. Fig. 5, Fotografía del zorro en la Reserva Natural del Volcán Azufral, Nariño (Colombia); Fig. 6, distribución potencial del zorro andino (color negro) con las localidades (L) de entrenamiento y evaluación en Colombia y Ecuador; Fig. 7, distribución potencial en Colombia. Los departamentos de Nariño y Putumayo presentan frontera al sur con la República de Ecuador. DZ: Distribución del zorro reportada por la UICN.

Tab. II. Área distribución potencial (DP) y área disponible (AD) para el zorro andino. Se incluyen los porcentajes (\%) de pérdida del área de distribución potencial y la cantidad de área habitable $(\mathrm{AH})$ que se encuentra dentro del Sistema Nacional de Áreas Protegidas de Colombia (SINAP) Asterisco $(*)=$ kilómetros cuadrados $\left(\mathrm{km}^{2}\right)$.

\begin{tabular}{lcccccc}
\hline Departamento & $\begin{array}{c}\text { Área distribución } \\
\text { potencial (DP)* }\end{array}$ & $\begin{array}{c}\text { Área Disponible } \\
(\mathrm{AD}) *\end{array}$ & $\begin{array}{c}\text { Perdida de área } \\
(\mathrm{DP}-\mathrm{AD})^{*}\end{array}$ & \% Perdida & $\begin{array}{c}\text { AH Protegida en } \\
\text { SINAP* AH Protegida } \\
\text { SINAP }\end{array}$ \\
\hline Nariño & 9572 & 5076 & 4496 & 47.0 & 442 & 8.7 \\
Cauca & 3268 & 2336 & 932 & 28.5 & 447 & 19.1 \\
Huila & 1361 & 1144 & 217 & 15.9 & 772 & 67.5 \\
Putumayo & 399 & 321 & 78 & 19.5 & 81 & 25.2 \\
Total Colombia & 14600 & 8877 & 5723 & 39.2 & 1742 & 19.6 \\
\hline
\end{tabular}

páramo de Las Papas en el Huila (Hershkovitz, 1957), sin embargo se deben fortalecer los monitoreos de la especie para reforzar la validación del mapa, especialmente para la zona nororiental de los Andes del departamento de Nariño y sur de los departamentos del Cauca y Huila de donde se desconocen registros verificados para la especie, aunque el modelo indica que el ambiente es idóneo. Esta es una zona contigua a las localidades de registro de L. culpaeus en Colombia, sin barreras naturales o accidentes geográficos que limiten su distribución, por lo que no existe ninguna razón aparente para dudar de la presencia de la especie en el área. Sin embargo, en este sector no se han realizado estudios de campo. Más al norte se espera que el zorro andino esté ausente ya que para el PNN Puracé estudios recientes mediante cámaras trampa no detectaron evidencia de su presencia, aunque si detectaron otras especies de carnívoros (HERNÁNDEZ-GuZMÁN et al.,
2011; DUQUE LóPEZ et al., 2013). La ausencia de registros del zorro andino al norte del páramo de Las Papas en el departamento de Cauca y Huila, quizás esté relacionada con la barrera natural causada por el río Magdalena que marca una dicotomía entre las cordilleras Oriental y Central conformada por áreas estrechas y lo suficientemente profundas para evitar el paso de mamíferos (HershKovitz, 1982).

En el caso de la distribución potencial de L. culpaeus, esta se encuentra de manera fragmentada dentro de 22 áreas protegidas, las cuales cuentan con legislación especial, por lo que la calidad de hábitat tiende a mantenerse. Entre ellas sobresalen algunas de mayor tamaño e importancia para la conservación del patrimonio natural del país (PNN Complejo Volcánico Doña Juana Cascabel, Reserva Forestal Protectora Nacional Rivera Río Mocoa, RFPN Hoya de los ríos Bobo y Buesaquillo, Santuario de Flora y Fauna Galeras) y otras de 


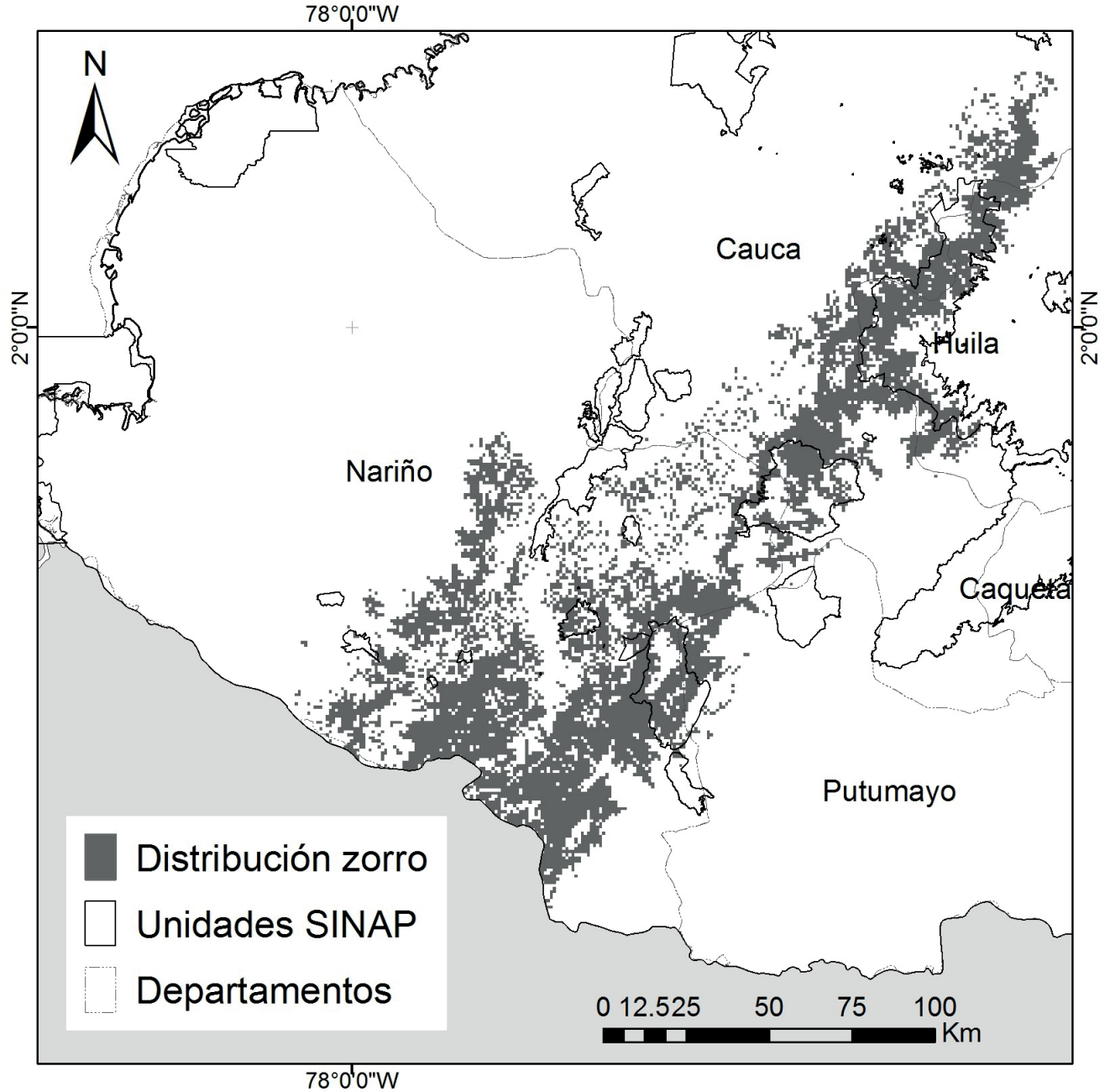

Fig. 8. Distribución del zorro andino considerando cobertura vegetal disponible y áreas protegidas de Colombia.

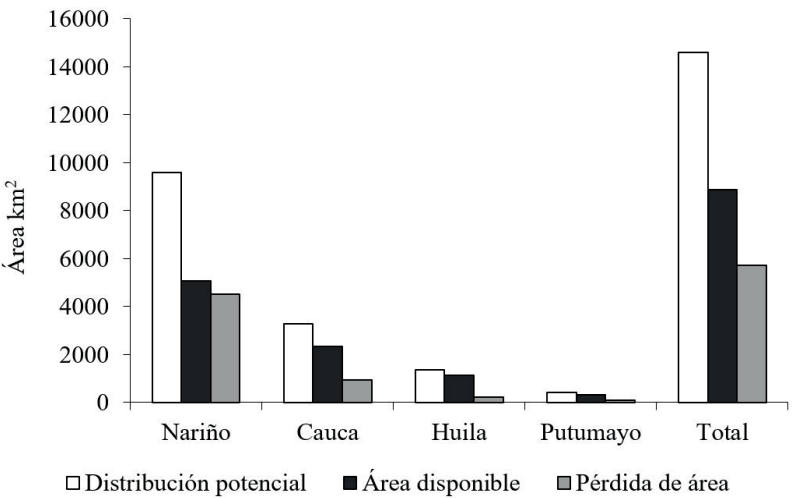

Fig. 9. Cambios del área de distribución del zorro andino en Colombia. La pérdida de área de distribución potencial (DP) fue calculada de la siguiente forma: (DP-AD).

importancia regional (Laguna La Cocha y Cerro Patascoy, Parque Natural Regional Serranía de Minas, PNR Corredor de Los Guacharos, Reserva Forestal Protectora Regional Área Circundante Volcán Azufral, Reserva Privada Pueblo Viejo).

En Colombia las poblaciones del zorro andino han sido incluidas en la categoría de amenaza Vulnerable - VU (MADT, 2014), justificada en la transformación del hábitat y la cacería por retaliación como causas de su disminución poblacional (JoRGENSON et al., 2006). Sin embargo, no existen estudios puntuales sobre los temas antes mencionados a nivel nacional. La información disponible en literatura y nuestras observaciones muestran que estos dos aspectos pueden estar escasamente afectando la especie en Colombia. Por ejemplo, la transformación de hábitat, aunque debe afectar a la especie, parece ser aprovechada por el zorro. Este hecho está soportado por los registros recientes en áreas intervenidas, donde individuos de la especie son detectados constantemente en zonas de asentamientos humanos rurales y potreros. Para otros países se ha estimado que la especie incluye hasta en un $85 \%$ de su ámbito de acción a zonas cercanas a habitaciones humanas (SALVATORI et al., 1999) y es generalista en su dieta (WALKER et al., 2007) por lo que puede subsistir sin depender de animales domésticos. Con respecto a la cacería por retaliación, es necesario resaltar que su impacto sobre la especie no fue directamente evaluado en este trabajo, sin embargo en las zonas visitadas otros carnívoros (e.g. el oso de anteojos Tremarctos ornatus) sufren mayor presión. Además, especies como el venado 
(Mazama rufina) y roedores de gran tamaño (e.g. Cuniculus taczanowskii) son fuertemente cazados para consumo, lo cual se evidencia en la cantidad de fragmentos de pieles o cráneos que se pueden registrar en la zona para estas especies, mientras que evidencia secundaria del zorro andino es difícil de obtener debido a que aparentemente la especie no es usada localmente.

Es claro que el escaso conocimiento sobre la especie en Colombia y la falta de información obtenida directamente de actividades de campo, son algunas de las razones para categorizaciones de amenaza previas tan altas, lo que se respalda con nuestros resultados. A nivel nacional, existen antecedentes para otros vertebrados donde se ha mostrado que las categorías de amenaza se basan más en criterios anecdóticos que en desarrollo de campo y evaluaciones poblacionales (v.g. Mueses-Cisneros, 2005). Este parece ser el caso para el zorro andino en Colombia donde aspectos básicos de la biología o distribución de la especie no fueron analizados en la categorización previa (JORGENSON et al., 2006) y la especie no cumple con los criterios para ser incluida en la categoría VU. A parte de los problemas asociados a la falta de datos para la categorización anterior (JORGENSON et al., 2006), el intervalo de tiempo trascurrido desde la primera asignación para Colombia, justifican la presente reevaluación y transferencia de categoría a Casi Amenazada (NT), que se apoya en datos nacionales recientes de la especie.

Por otra parte, aún persiste la necesidad de desarrollar nuevas investigaciones sobre el estado de las poblaciones del zorro andino, así como sus usos, amenazas, historia natural y ecología (SuÁrEZ-CASTRo et al., 2015). Esta necesidad de estudios se incrementa al considerar que Colombia constituye el límite boreal en la distribución de la especie. Para esto, el uso de entrevistas y observaciones de campo pueden emplearse para analizar las amenazas y presiones que sufre la especie en la actualidad. Así mismo, la implementación de nuevas técnicas como el uso de trampas cámara que se ha probado exitosas para los Andes del Ecuador (e. g. ZAPATA-RÍOS \& BRANCH, 2016), puede ser de gran utilidad para llenar estos vacíos de información.

A la fecha se están realizando esfuerzos para la conservación del zorro andino en el suroccidente de Nariño, donde se ha propuesto un Plan de Conservación para la especie en el Parque Natural Regional Páramo de Paja Blanca (CALDERón et al., 2010). Esto sería un primer avance hacia la conservación de la especie, ya que si se considera los resultados obtenidos, Nariño presenta la mayor área disponible para la especie, pero también la mayor área intervenida. Debido a que los Andes del país representan uno de los territorios más transformados por actividades productivas como la agricultura y la ganadería (RODRíGUEZERASO et al., 2010), también se debe realizar actividades enfocadas a preservar hábitats y la conectividad de estos en estas zonas que faciliten la conservación del zorro andino, que también se ha observado en zonas intervenidas en el departamento de Nariño.
Agradecimientos. Christian Cabrera del Grupo de Investigación Ecología Evolutiva (GIEE) de la Universidad de Nariño y la Asociación GAICA facilitaron la fotografía e información de algunos registros en Nariño. A Jhon Jairo Calderón y Oswaldo Arcos del Museo PSO-CZ de la Universidad de Nariño por permitir acceso a la colección. A Luis Lasso y Silvia Montenegro por su acompañamiento durante expediciones en campo. HER-C agradece a las becas UQ cent, UQI y GSITA de la Universidad de Queensland por el apoyo brindado. EAN-U agradece al Posgrado en Ciencias de la Universidad Nacional Autónoma de México (UNAM) y al Consejo Nacional de Ciencia y Tecnología (CONACyT) por el apoyo brindado para estudios de Doctorado (Beca 262582) durante el desarrollo de esta investigación.

\section{REFERENCIAS}

Albuja, L. 2007. Biología y ecología del venado de cola blanca (Odocoileus virginianus ustus Gray, 1874) en los Páramos de Oyacachi-Papallacta y Antisana, Ecuador. Politécnica 27:34-57.

Anderson, R. P. \& GonZalez, I. 2011. Species-specific tuning increases robustness to sampling bias in models of species distributions: an implementation with Maxent. Ecological Modelling 222:2796-2811.

Araúso, M. B. \& Peterson, A.T. 2012. Uses and misuses of bioclimatic envelope modeling. Ecology 93:1527-1539.

Arcos, R. 2010. Riqueza y abundancia relativa de mamíferos en la Cordillera Oriental Yacuambi, en el suroriente ecuatoriano. Boletín Técnico 9:147-161.

Barve, N. 2008. Tool for Partial-ROC. Version 1. Lawrence, KS: Biodiversity Institute. Disponible en $<$ http://kuscholarworks.ku.edu/ dspace/handle/1808/10059>. Visitado 15 Agosto 2014.

Barve, N.; Barve, V.; JimeneZ-Valverde, A.; LiRa-Noriega, A.; Maher, S. P.; Peterson, A. T.; Soberón, J. \& Villalobos, F. 2011. The crucial role of the accessible area in ecological niche modeling and species distribution modeling. Ecological Modelling 222:1810-1819.

Calderón, J. J.; Lasso, L. \& Montenegro-Muñoz, S. 2010. Plan de conservación del lobo colorado o "morito" (Lycalopex culpaeus, Molina 1782) en el Parque Natural Regional Páramo de Paja Blanca. Informe técnico. Nariño, Corporación Autónoma Regional de Nariño y la Asociación Grupo de Amigos para la Investigación y Conservación de las Aves - GAICA. 62p.

Castro, I. \& Roman, H. 2000. Evaluación ecológica rápida de la mastofauna en el Parque Nacional Llanganates $I n$ : VÁZQUEZ, A.; Larrea, M. \& SuÁrez, L. eds. Biodiversidad en el Parque Nacional Llanganates: un reporte de las evaluaciones ecológicas y socioeconómicas rápidas. Quito, EcoCiencia, Ministerio del Ambiente, Herbario Nacional del Ecuador, Museo Ecuatoriano de Ciencias Naturales e Instituto Internacional de Reconstrucción Rural, p. 129-146.

Chan, L. M.; Brown, J. L. \& Yoder, A. D. 2011. Integrating statistical genetic and geospatial methods brings new power to phylogeography. Molecular Phylogenetics and Evolution 59:523-537.

Cossíos, E. D.; Alcazár, P.; Fajardo, U.; Chávez, K.; Alfaro-Shigueto, J.; Cárdenas-Alayza, S.; Valqui, J.; Montero, F. G.; Lescano, J.; Quevedo, M.; Vivar, E.; Leite, R.; Ledesma, K.; Medina, C.; Maffei, L.; Amanzo, J.; Chávez, C.; Enciso, A.; García, M. A.; Mangel, J. C.; Mendoza, J.A.; Rojas, G.; Silva, L.; Villegas, L.; Williams, R. S. R.; ZÚÑIGA, A.; CRUZ, A.; IMARPE; RUIZ, E. \& DGFFSS. 2012. El orden Carnivora (Mammalia) en el Perú: Estado del conocimiento y prioridades de investigación para su conservación. Revista Peruana de Biología 19(1):17-26.

Cuyckens, G. A. E.; Perovic, P. G. \& Cristobal, L. 2015. How are wetlands and biological interactions related to carnivore distributions at high altitude? Journal of Arid Environments 115:14-18.

de Castro Pena, J. C.; Kamino, L. H. Y.; Rodrigues, M.; Mariano-Neto, E. \& DE Siqueira, M. F. 2014. Assessing the conservation status of species with limited available data and disjunct distribution. Biological Conservation 170:130-136.

de Vriess, T.; Black, J.; Solís, C. \& Hernández, C. 1983. Historia natural del curiquingue. En los páramos de Antisana y Cotopaxi en el Ecuador. Quito, Ediciones de la Universidad Católica. 83p.

Defourny, P. \& VAN Bogaert, E. 2014. Land cover ESACCI-LC-L4LCCS-Map-300m-P5Y-2010-v1.4. Disponible en $<\mathrm{http}: / /$ maps.elie. ucl.ac.be/CCI/viewer/download.php>. Visitado 15 Enero 2015. 
Duque lópez, S.; Abud, M.; Calero, H. \& Valderrama, S. 2013. Cameratrap Records of Mountain Tapir in Puracé National Park, Colombia. Tapir Conservation 22:5-8.

HANLEY, J. A. \& MCNEIL, B. J. 1982. The meaning and use of the area under a Receiver Operating Characteristic (ROC) curve. Radiology 143:29-36.

HeIberger, R. 2015. Paquete HH: Statistical analysis and data display: Heiberger and Holland. Vienna, R Foundation for statistical computing. Disponible em <http://www.R-project.org/>. Visitado 30 Enero 2015.

Hernández-Guzmán, A.; PAyán, E. \& Monroy-Vilchis, O. 2011. Hábitos alimentarios del Puma concolor (Carnivora: Felidae) en el Parque Nacional Natural Puracé, Colombia. Revista de Biología Tropical 59:1285-94.

Hershkovitz, P. 1957. A synopsis of the wild dogs of Colombia. Novedades Colombianas, Museo de Historia Natural, Universidad del Cauca 3:157-162

Hershkovitz, P. 1982. Neotropical deer (Cervidae) Part 1. Pudus genus Pudu Gray. Fieldiana, Zoology, New series 11:1-86.

Hijmans, R. J.; van Etten, J.; Mattiuzzi, M.; Sumner, M.; Greenberg, J.; Perpinan, O.; Bevan, A.; Racine, E. \& Shortridge, A. 2005. Paquete RASTER. Vienna, R Foundation for Statistical Computing. Disponible en $<$ http://cran.r-project.org/web/packages/raster/index. html>. Visitado 24 Abril 2014.

Jiménez, J. E.; Lucherini, M. \& Novaro, A. J. 2008. Pseudalopex culpaeus. In: UICN, 2013. UICN Red List of Threatened Species. Version 20132. Disponible en $<$ http://UICNredlist.org >. Visitado 26 Abril 2014.

Jorgenson, J. P.; Rodríguez-Mahecha, J. V.; Constantino, E. \& BARRERA DE Jorgenson, A. 2006. Lobo colorado Lycalopex culpaeus. In: Rodríguez- Mahecha, J. V.; Alberico, M.; Trujillo, F. \& Jorgenson, J. eds. Libro rojo de los mamíferos de Colombia. Bogotá, Conservación Internacional Colombia, Ministerio de Ambiente, Vivienda y Desarrollo Territorial, p 237-241.

MADT - Ministerio del Ambiente y Desarrollo Sostenible. 2014. Resolución No. 0192, "Por la cual se establece el listado de las especies silvestres amenazadas de la diversidad biológica colombiana que se encuentran en el territorio nacional, y se dictan otras determinaciones". Bogotá, 10 Febrero 2014, p. 1-36.

Morato, R. G.; de Barros, K. M. P. M.; de Paula, R. C. \& de Campos, C. B. 2014. Identification of priority conservation areas and potential corridors for jaguars in the CDPtinga Biome, Brazil. PloS One 9(4):e92950.

Mueses-Cisneros, J. J. 2005. Crítica a la asignación de la categoría de amenaza de Gastrotheca ruizi (Amphibia: Anura: Hylidae). Revista de la Academia Colombiana de Ciencias Exactas, Físicas y Naturales 29:303-308.

NARvÁEZ, C. \& CiSNERos, R. 2013. Análisis de estacionalidad y abundancia relativa de oso de anteojos (Tremarctos ornatus), lobo de páramo (Lycalopex culpaeus) y danta de montaña (Tapirus pinchaque) en los páramos del Parque Nacional Podocarpus. In: TIRIRA, D. ed. Memorias del I Congreso Latinoamericano de Tapires y II Congreso Ecuatoriano de Mastozoología. Puyo Pastaza- Ecuador. Quito, Publica asesores Cía. Ltda, p. 78-79.

Narváez, C.; Salazar, M.; Valle, D.; Loaiza, C. \& Cinsneros, R. 2012. Mammals of Southern Ecuador: mountains (Loja). Chicago, The Field Museum. Disponible en $<\mathrm{http}$ ://fm2.fieldmuseum.org/plantguides/ guide_pdfs/403\%20Mamiferos\%20Comunes.pdf.> Visitado 16 Agosto 2014.

Novaro, A. J. 1997. Pseudalopex culpaeus. American Society of Mammalogists. Mammalian Species 558:1-8.

Olson, D. M.; Dinerstein, E.; Wikramanayake, E. D.; Burgess, N. D.; Powell, G. V. N.; Underwood, E. C.; D’Amico, J. A.; Strand, H. E.; Morrison, J. C.; Loucks, C. J.; Allnutt, T. F.; LamoreuX, J. F.; Ricketts, T. H.; Itoua, I.; Wettengel, W. W.; Kura, Y.; Hedao, P. \& KASSEM, K. 2001. Terrestrial ecoregions of the world: a new map of life on Earth. BioScience 51:933-938.

Ortega-Andrade, H. M.; Prieto-Torres, D. A.; GómeZ-Lora, I.; LizCANo, D. J. 2015. Ecological and geographical analysis of the distribution of the Mountain Tapir (Tapirus pinchaque) in Ecuador: Importance of protected areas in future scenarios of global warming. PLoS One 10(3):e0121137.

Parques Nacionales Naturales de Colombia. 2014. Datos abierto, información geográfica SINAP. Disponible en <http://www. parquesnacionales.gov.co/portal/servicio-al-ciudadano/datos-abiertos/>. Visitado 30 Enero 2015.
Peterson, A. T. 2012. Niche modeling: model evaluation. Biodiversity Informatic 8:41.

Peterson, A. T.; Papeş, M. \& Soberón, J. 2008. Rethinking receiver operating characteristic analysis applications in ecological niche modeling. Ecological Modelling 213:63-72.

Peterson, A. T.; Soberón, J.; Pearson, R. G.; Anderson, R. P.; NaKamura, M.; Martinez-Meyer, E. \& Araújo, M. B. 2011. Ecological niches and geographical distributions. Princeton, Princeton University Press. 329p.

Phillips, S. J.; Anderson, R. P. \& Schapire, R. E. 2006. Maximum entropy modelling of species geographic distributions. Ecological Modelling 190:231-259.

PhILliPs, S. J. \& DUDIK, M. 2008. Modelling of species distribution with Maxent: new extension and a comprehensive evaluation. Ecography 31:161-175.

Pozo, W. \& Trujillo, F. 2005. Lista anotada de la fauna de la Laguna Loreto, Reserva Ecológica Cayambe Coca, Ecuador. Boletín Técnico 5:29-43.

R Development CoreTeAm. 2014. R: a language and environment for statistical computing. Vienna, R Foundation for Statistical Computing.

Ramírez-Chaves, H. E.; Chaves-Salazar, J. M. \& Mendoza-Escobar, R.H. 2013. Nuevo registro del Lobo de páramo Lycalopex culpaeus (Mammalia: Canidae) en el Suroccidente de Colombia con notas sobre su distribución en el país. Acta Zoológica Mexicana (n.s.) 29:412-422.

RamíreZ-Chaves, H. E. \& Noguera-Urbano, E. A. 2010. Lista preliminar de los mamíferos (Mammalia: Theria) del departamento de Nariño, Colombia. Biota Colombiana 11:117-140.

Rodríguez-Eraso, N.; Pabón-Caicedo, J. D.; Bernal-Suárez, N. R. \& Martínez-Collantes, J. 2010. Cambio climático y su relación con el uso del suelo en los Andes colombianos. Bogotá, Instituto de Investigación de Recursos Biológicos Alexander von Humboldt, Universidad Nacional de Colombia y Departamento Administrativo de Ciencia, Tecnología e Innovación. 80p

Rodríguez-Soto, C.; Monroy-Vilchis, O.; Maiorano, L.; Boitani, L.; Faller, J. C.; Briones, M. A.; Nuñez, R.; Rosas-Rosas, O.; Ceballos, G. \& Falcucci, A. 2011. Predicting potential distribution of the jaguar (Panthera onca) in Mexico: identification of priority areas for conservation. Diversity and Distributions 17:350-361.

Ruiz-García, M.; Rivas-SÁnchez, D. \& Linchilín-OrTiz, N. 2013. Phylogenetic relationships among four putative taxa of foxes of the Pseudalopex Genus (Canidae, Carnivora) and molecular population genetics of Ps. culpaeus and Ps. sechurae, In: RUIZ-GARCÍA, M. \& Shostell, J. M. eds. Molecular population genetics, evolutionary biology and biological conservation of the Neotropical carnivores. New York, Nova Publishers, p. 1-31.

Salvatori, V.; Vaglio-Laurin, G.; Meserve, P.; Boitani, L. \& Campanella, A. 1999. Spatial organization, activity, and social interactions of Culpeo foxes (Pseudalopex culpaeus) in North-Central Chile. Journal of Mammalogy 80:980-985.

Sillero-Zubiri, C. 2009. The Canidae. In: Wilson, D. E. \& Mittermeier, R. A. eds. The Handbook of the Mammals of the World 1. Carnivora. Barcelona, Lynxs Edicions asociada con Conservation International y UICN, p. 352-446

Solari, S.; Muñoz-Saba, Y.; RodríGuez-Mahecha, J. V.; Defler, T. R.; Ramírez-Chaves, H. E. \& Trujillo, F. 2013. Riqueza, endemismo y conservación de los mamíferos de Colombia. Mastozoología Neotropical 20:301-365

SuÁrez-Castro, A. F. \& Ramírez-Chaves, H. E. eds. 2015. Los carnívoros terrestres y semiacuáticos continentales de Colombia. Guía de campo. Bogotá, Universidad Nacional de Colombia. 214p.

Suárez-Castro, A. F.; Pinto-Sarmiento, I. D. \& García-Villalba, J. E. 2015. Familia Canidae. In: SuÁrez-CAStro, A. F. \& Ramírez-Chaves, H. E. eds. Los carnívoros terrestres y semiacuáticos continentales de Colombia. Guía de campo. Bogotá, Universidad Nacional de Colombia, p. 56-77.

TIRIRA, D. 2010. Mamíferos ecuatorianos en museos de historia natural y colecciones científicas II. El Museo Argentino de Ciencias Naturales "Bernardino Rivadavia”. Boletín Técnico 9:111-133.

Tirira, D. G. ed. 2011. Libro Rojo de los mamíferos del Ecuador. 2ed. Publicación Especial sobre los mamíferos del Ecuador 8. Quito, Fundación Mamíferos y Conservación, Pontificia Universidad Católica del Ecuador y Ministerio del Ambiente del Ecuador. 398p. 
Trujillo, J. \& Trujillo, F. G. 2007. Alimentación del Lobo (Lycalopex culpaeus) en el Bosque Protector Jerusalén, Guayllabamba-Ecuador Politécnica 27:68-75.

UICN. 2012a. Categorías y Criterios de la Lista Roja de la UICN: Versión 3.1. Segunda edición. Gland, Suiza y Cambridge, UICN. Originalmente publicado como UICN Red List Categories and Criteria: Version 3.1. Second edition. (Gland, Switzerland and cambridge, UK: UICN, 2012). UICN. 34p.

UICN. 2012b. Directrices para el uso de los Criterios de la Lista Roja de la UICN a nivel regional y nacional: Versión 4.0 . Gland, Suiza y Cambridge, UICN. Originalmente publicado como Guidelines for Application of UICN Red List Criteria at Regional and National Levels: Version 4.0. (Gland, Switzerland and cambridge, UK: UICN, 2012). UICN. 43 p.
Walker, R.; Novaro, A.; Perovic, P.; Palacios, R.; Donadio, E.; Lucherini, M.; PiA, M. \& Lopez, M. 2007. Diets of three species of Andean carnivores in high-altitude deserts of Argentina. Journal of Mammalogy 88:519-525.

ZAPATA-Ríos, G. \& BRANCH, L. C. 2016. Altered activity patterns and reduced abundance of native mammals in sites with feral dogs in the high Andes. Biological Conservation 193:9-16.

Zunino G. E.; Vaccaro, O. B.; Canevari, M. \& Gardner, A. L. 1995. Taxonomy of the genus Lycalopex (Carnivora: Canidae) in Argentina. Proceedings of the Biological Society of Washington 108:729-747.

Zuur, A. F.; Ieno, E. N. \& Elphick, C. S. 2010. A protocol for data exploration to avoid common statistical problems. Methods in Ecology and Evolution 1:3-14.

Apéndice 1. Localidades empleadas para la obtención del modelo de distribución del zorro andino. Se proveen las coordenadas geográficas en grados decimal, datum WGS84. El símbolo (*) indica las localidades usadas en el entrenamiento del modelo, las restantes fueron usadas en la evaluación externa del modelo. Fuentes: MaNIS, Mammal Networked Information System; Trabajo de campo, obtenidos durante exploraciones en los Páramos de Nariño (Colombia); GIEE, Grupo de Investigación de Ecología Evolutiva, Universidad de Nariño (Christian Cabrera, com. pers.).

\begin{tabular}{|c|c|c|c|c|}
\hline País & Longitud & Latitud & Departamento/Localidad & Fuente \\
\hline Colombia* & -77.65 & 1.06 & Nariño/Guachucal & GIEE \\
\hline Colombia* & -77.67 & 1.03 & Nariño/Guachucal & Trabajo de campo \\
\hline Colombia* & -77.69 & 1.04 & Nariño/Guachucal & Trabajo de campo \\
\hline Colombia* & -77.68 & 1.05 & Nariño/Guachucal & Trabajo de campo \\
\hline Colombia* & -77.27 & 1.22 & Nariño/Pasto & MaNIS (FMNH 85822) \\
\hline Colombia* & -77.23 & 1.02 & Nariño/Pasto & GIEE \\
\hline Colombia & -77.12 & 1.20 & Nariño/Pasto & GIEE \\
\hline Colombia* & -77.25 & 1.43 & Nariño/Chachagüi & RAMÍREZ- ChaVES et al., 2013 \\
\hline Colombia & -77.56 & 1.07 & Nariño/Guachucal & Trabajo de campo \\
\hline Colombia & -77.57 & 0.95 & Nariño/Gualmatan & GIEE \\
\hline Colombia & -77.68 & 1.08 & Nariño/Guachucal & GIEE \\
\hline Colombia & -77.53 & 0.67 & Nariño/Ipiales & RAMÍREZ-CHAVES et al., 2013 \\
\hline Colombia* & -77.50 & 0.88 & Nariño/Puerres & Ramírez- Chaves \& Noguera-Urbano, 2010 \\
\hline Colombia* & -77.61 & 1.03 & Nariño/Sapuyes & Ramírez- Chaves \& Noguera-Urbano, 2010 \\
\hline Colombia* & -77.30 & 1.07 & Nariño/Tangua & GIEE \\
\hline Colombia* & -77.74 & 1.19 & Nariño/Túquerres & GIEE \\
\hline Colombia* & -77.71 & 1.16 & Nariño/Túquerres & GIEE \\
\hline Colombia* & -77.09 & 1.16 & Nariño/Túquerres & GIEE \\
\hline Colombia* & -77.85 & 1.17 & Nariño/Túquerres & GIEE \\
\hline Colombia & -77.82 & 1.18 & Nariño/Túquerres & GIEE \\
\hline Colombia & -77.72 & 1.18 & Nariño/Túquerres & GIEE \\
\hline Colombia & -77.63 & 1.16 & Nariño/Túquerres & GIEE \\
\hline Colombia & -77.75 & 1.17 & Nariño/Túquerres & GIEE \\
\hline Colombia & -77.78 & 1.16 & Nariño/Túquerres & GIEE \\
\hline Colombia & -77.80 & 1.16 & Nariño/Túquerres & GIEE \\
\hline Ecuador & -79.22 & -3.17 & Azuay/Bestion, Girón & MaNIS voucher \\
\hline Ecuador* & -79.00 & -1.47 & Bolívar/Guaranda & MaNIS (AMNH M-67087) \\
\hline Ecuador* & -79.20 & -3.98 & Loja & NARVÁEZ et al., 2012 \\
\hline Ecuador & -78.97 & -4.36 & Loja/Páramos Parque Nacional Podocarpus & NARVÁEZ \& CiSNEROS, 2013 \\
\hline Ecuador* & -78.16 & -0.53 & Napo, Cerro Antisana & TIRIRA, 2010 \\
\hline Ecuador* & -78.25 & -0.22 & Napo/Tungurahua & MaNIS (FMNH 47053) \\
\hline Ecuador* & -78.07 & -0.08 & Pichincha/Alchipichi & RuIZ-GARcía et al., 2013 \\
\hline Ecuador & -78.27 & -0.10 & Pichincha/Cayambe, Cangaua & MaNIS voucher \\
\hline Ecuador* & -78.47 & -0.62 & Pichincha/Cotopaxi & MaNIS (AMNH M-66735) \\
\hline Ecuador* & -78.16 & -0.38 & Pichincha/Laguna Loreto & Pozo \& Trujillo, 2005 \\
\hline Ecuador & -78.60 & -0.17 & Pichincha/Pichincha & TIRIRA, 2010 \\
\hline Ecuador* & -78.15 & -0.45 & Pichincha/ Quito & MaNIS (AMNH M-36454) \\
\hline Ecuador & -78.31 & -0.45 & Pichincha/Quito, Antisanilla & MaNIS voucher \\
\hline Ecuador & -78.13 & -0.30 & Pichincha/Quito, Oyacachi - Papallacta & Albuja, 2007 \\
\hline Ecuador & -78.36 & 0.00 & Pichincha/Quito, Bosque Guayabamba & Trujillo \& Trujillo, 2007 \\
\hline Ecuador* & -78.16 & -0.55 & Pichincha-Napo/Páramo de Antisana & DE VRIESS et al., 1983 \\
\hline Ecuador* & -78.25 & -1.15 & Tungurahua/Rio Ana Tenorio & Castro \& Roman, 2000 \\
\hline Ecuador* & -78.32 & -1.09 & Tungurahua/Laguna de Pisayambo & CASTRO \& ROMAN, 2000 \\
\hline Ecuador* & -79.08 & -3.62 & Zamora-Chinchipe/Yacuambi & Arcos, 2010 \\
\hline
\end{tabular}

